\title{
Appendix for Regularized Brain Reading with Shrinkage and Smoothing
}

\section{Appendix A: Small Area model and Gibbs sampler}

Here, we specify the details of the Gibbs sampler for the hierarchical Bayesian small-area model described in $\S 2.3$. Recall that $A(v)$ is the area which voxel $v$ resides in, and the $V(a)$ the set of all voxels in area $a$.

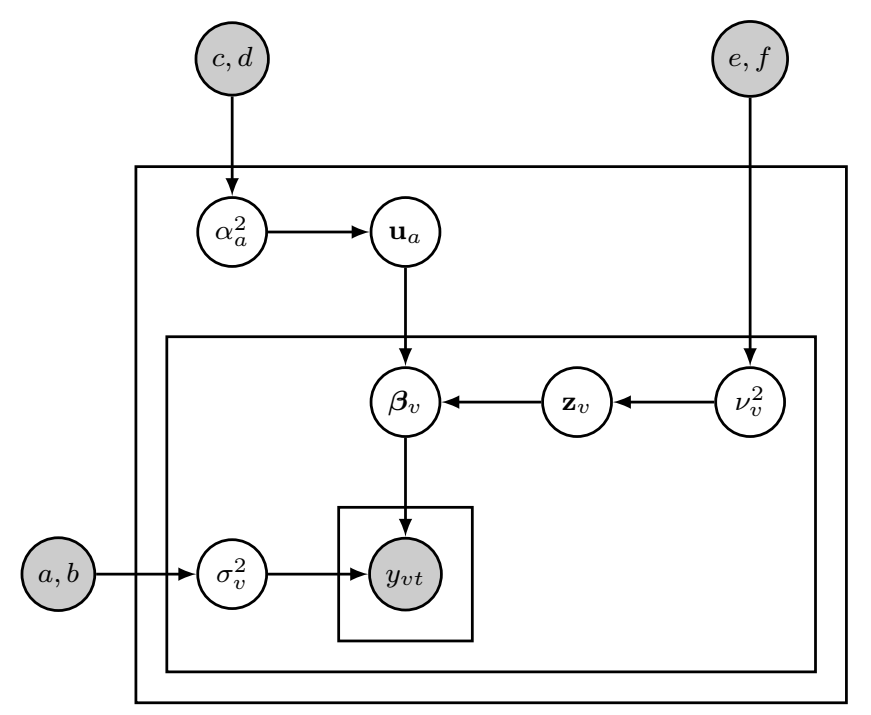

Fig 1: Graphical model representation of the small-area model of $\S 2.3$.

The joint distribution can be written as

$$
\begin{aligned}
P\left(\mathbf{Y}, \mathbf{U}, \beta, \alpha^{2}, \nu^{2}, \sigma^{2}\right) & =\prod_{a} \mathcal{I} \mathcal{G}\left(\alpha_{a}^{2} \mid c, d\right) \times \prod_{v} \mathcal{I} \mathcal{G}\left(\nu_{v}^{2} \mid e, f\right) \times \prod_{v} \mathcal{I} \mathcal{G}\left(\sigma_{v}^{2} \mid a, b\right) \\
\times & \prod_{a} \mathcal{N}\left(\mathbf{u}_{\mathbf{a}} \mid 0, \alpha_{a}^{2} \mathbf{I}\right) \times \prod_{v} \mathcal{N}\left(\mathbf{z}_{v} \mid 0, \nu_{v}^{2} \mathbf{I}\right) \times \prod_{v} \prod_{t} \mathcal{N}\left(y_{v t} \mid\left(\mathbf{u}_{A(v)}+\mathbf{z}_{v}\right)^{\top} \mathbf{x}_{t}, \sigma_{v}^{2}\right) .
\end{aligned}
$$

We now derive the full conditionals from the joint distribution above. 


$$
\begin{aligned}
& P\left(\mathbf{z}_{v} \mid \ldots\right) \propto \mathcal{N}\left(\mathbf{z}_{v} \mid 0, \nu_{v}^{2} \mathbf{I}\right) \times \prod_{t} \mathcal{N}\left(y_{v t} \mid\left(\mathbf{u}_{A(v)}+\mathbf{z}_{v}\right)^{\top} \mathbf{x}_{\mathbf{t}}, \sigma_{v}^{2}\right) \\
& =\mathcal{N}\left(\mathbf{z}_{v} \mid \boldsymbol{\mu}_{\mathbf{z}_{v}}, \boldsymbol{\Sigma}_{\mathbf{z}_{v}}\right) \\
& \boldsymbol{\Sigma}_{\mathbf{z}_{v}}=\left(\frac{1}{\nu_{v}^{2}} \mathbf{I}+\frac{1}{\sigma_{v}^{2}} \mathbf{X}^{\top} \mathbf{X}\right)^{-1} \\
& \boldsymbol{\mu}_{\mathbf{z}_{v}}=\boldsymbol{\Sigma}_{\mathbf{z}_{v}} \times \frac{\left(\mathbf{X}^{\top} \mathbf{y}_{\mathbf{v}}-\mathbf{X}^{\top} \mathbf{X} \mathbf{u}_{A(v)}\right)}{\sigma_{v}^{2}} . \\
& P\left(\mathbf{u}_{a} \mid \ldots\right) \propto \mathcal{N}\left(\mathbf{u}_{\mathbf{a}} \mid 0, \alpha_{a}^{2} \mathbf{I}\right) \times \prod_{V(a)} \prod_{t} \mathcal{N}\left(y_{v t} \mid\left(\mathbf{u}_{A(v)}+\mathbf{z}_{v}\right)^{\top} \mathbf{x}_{\mathbf{t}}, \sigma_{v}^{2}\right) \\
& =\mathcal{N}\left(\mathbf{u}_{a} \mid \boldsymbol{\mu}_{\mathbf{u}_{a}}, \boldsymbol{\Sigma}_{\mathbf{u}_{a}}\right) \\
& \boldsymbol{\Sigma}_{\mathbf{u}_{a}}=\left(\frac{1}{\alpha_{a}^{2}} \mathbf{I}+\mathbf{X}^{\top} \mathbf{X} \sum_{V(a)} \frac{1}{\sigma_{v}^{2}}\right)^{-1} \\
& \boldsymbol{\mu}_{\mathbf{u}_{a}}=\boldsymbol{\Sigma}_{\mathbf{u}_{a}} \sum_{V(a)} \frac{\left(\mathbf{X}^{\top} y_{v}-\mathbf{X}^{\top} \mathbf{X} \mathbf{z}_{v}\right)}{\sigma_{v}^{2}} \\
& P\left(\sigma_{v}^{2} \mid \ldots\right) \propto \mathcal{I} \mathcal{G}\left(\sigma_{v}^{2} \mid a, b\right) \times \prod_{t} \mathcal{N}\left(y_{v t} \mid \boldsymbol{\beta}_{v}^{\top} \mathbf{x}_{\mathbf{t}}, \sigma_{v}^{2}\right) \\
& =\mathcal{I} \mathcal{G}\left(\sigma_{v}^{2} \mid a^{\prime}, b^{\prime}\right), \\
& a^{\prime}=\frac{2 a+T}{2} \\
& b^{\prime}=\frac{2 b+\left(\mathbf{y}_{v}-\mathbf{X} \boldsymbol{\beta}_{v}\right)^{\top}\left(\mathbf{y}_{v}-\mathbf{X} \boldsymbol{\beta}_{v}\right)}{2} . \\
& P\left(\alpha_{a}^{2} \mid \ldots\right) \quad \propto \mathcal{I} \mathcal{G}\left(\alpha_{a}^{2} \mid c, d\right) \times \mathcal{N}\left(\mathbf{u}_{\mathbf{a}} \mid 0, \alpha_{a}^{2} \mathbf{I}\right), \\
& =\mathcal{I} \mathcal{G}\left(\alpha_{a}^{2} \mid c^{\prime}, d^{\prime}\right) \\
& c^{\prime}=\frac{2 c+P}{2} \\
& d^{\prime}=\frac{2 d+\mathbf{u}_{a}^{\top} \mathbf{u}_{a}}{2} . \\
& P\left(\nu_{v}^{2} \mid \ldots\right) \propto \mathcal{I} \mathcal{G}\left(\nu_{v}^{2} \mid e, f\right) \times \mathcal{N}\left(\mathbf{z}_{v} \mid 0, \nu_{v}^{2} \mathbf{I}\right), \\
& =\mathcal{I} \mathcal{G}\left(\nu_{v}^{2} \mid e^{\prime}, f^{\prime}\right) \\
& e^{\prime}=\frac{2 e+P}{2} \\
& f^{\prime}=\frac{2 f+\mathbf{z}_{v}^{\top} \mathbf{z}_{v}}{2} .
\end{aligned}
$$



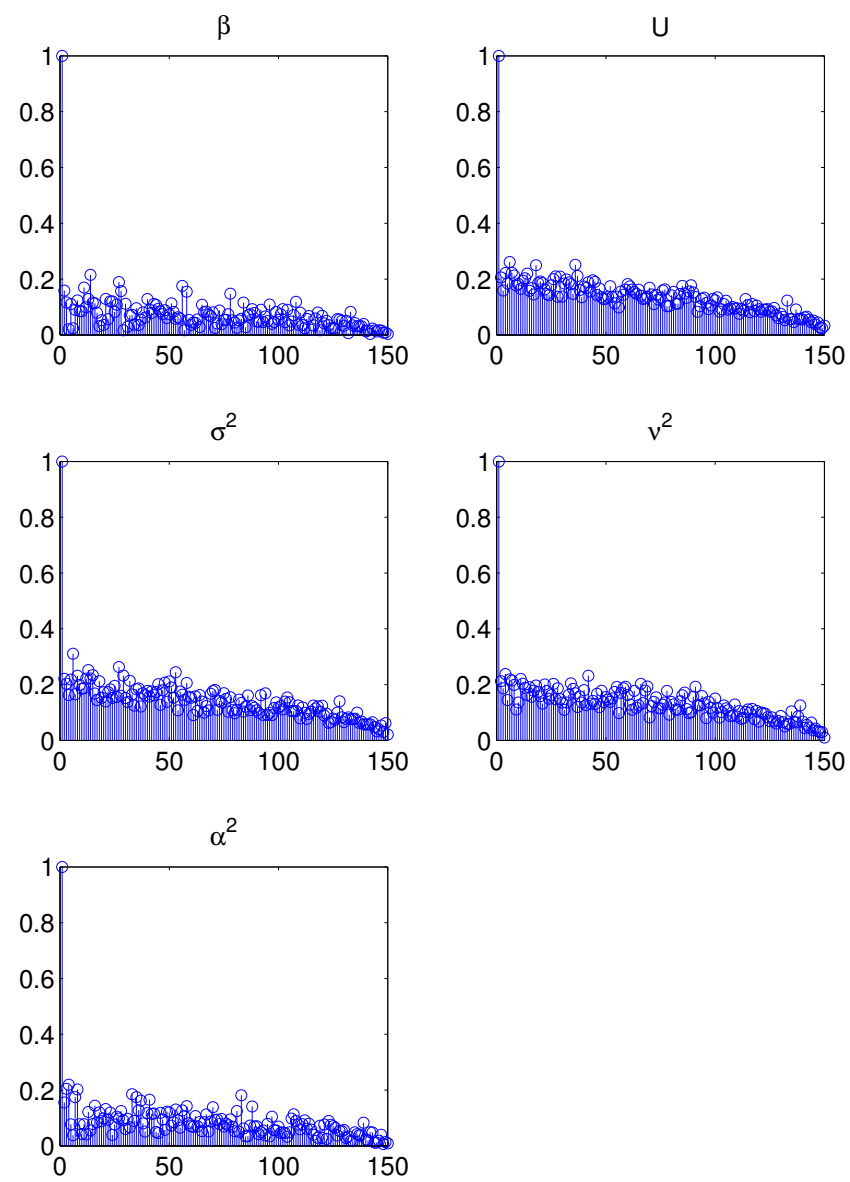

Fig 2: Maximum autocorrelation plots after burn-in and thinning. We used a thinning of 10 and a burn-in of 100 resulting in 150 samples. 


\section{Appendix B: The Marginal Prior of the SAE Model}

The small-area model has a Gaussian prior distribution $\mathbf{z}_{v} \mid \nu_{v}^{2} \sim \mathcal{N}\left(0, \nu_{v}^{2} \mathbf{I}\right)$ for the regression coefficients specific to voxel $v$. The voxel-specific variance has an inverse gamma prior distribution, where $\nu_{v}^{2} \sim \mathcal{I} \mathcal{G}(e, f)$. As mentioned in $\S 4.1$, this implies that the marginal prior distribution of $\mathbf{z}_{v}$ is a scaled $t$ distribution, where $\mathbf{z}_{v} /(f / e) \sim t_{2 e}$ (see ?, §3.3). This $t$ distribution approaches a Gaussian rather quickly as the number of degrees of freedom grows. Figure 3 shows the density obtained from $10^{4}$ draws from the hierarchical prior when $e=3$ (so that there are 6 degrees of freedom), along with the theoretical $t$ distribution, and the approximating Gaussian.

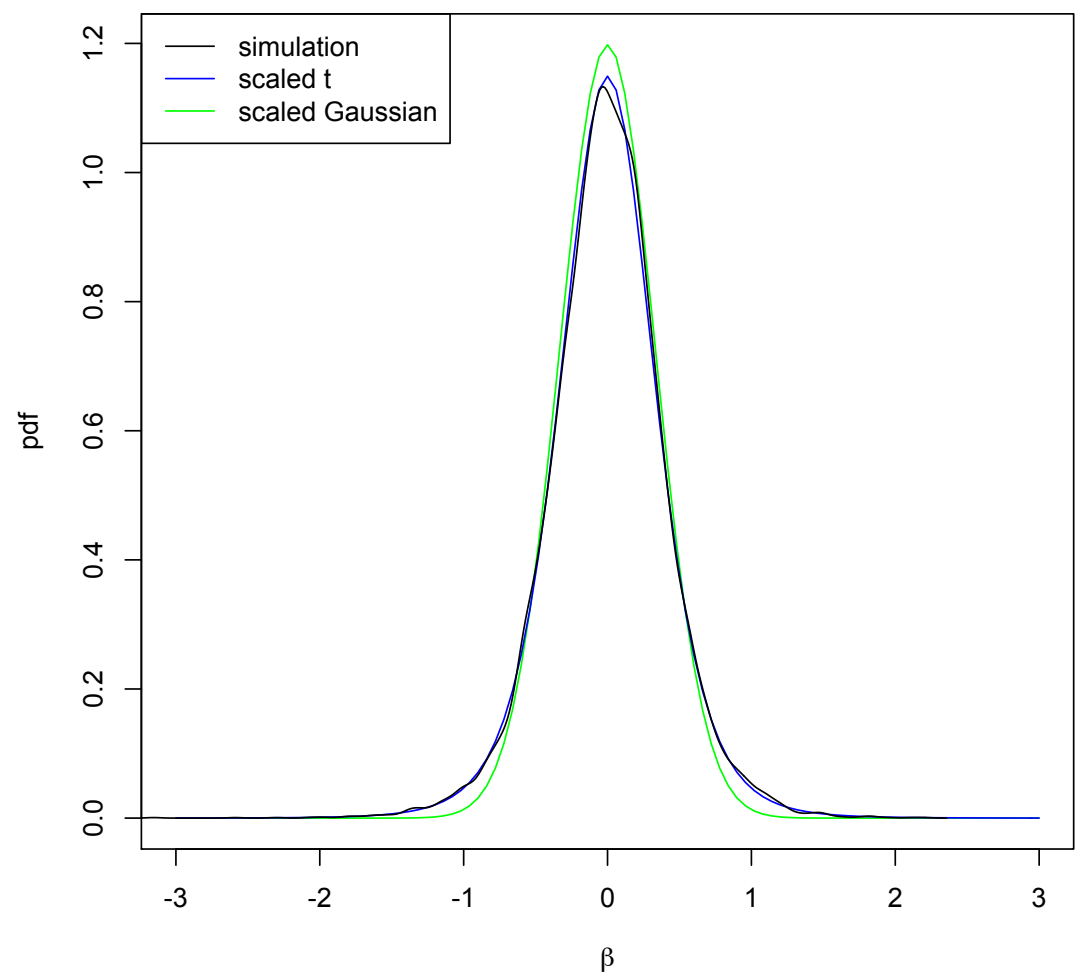

Fig 3: The SAE model's marginal prior distribution for regression coefficients, when the hyper-parameter $e=3$. The black line shows $10^{4}$ draws from the hierarchical prior, the blue the theoretical $t$ distribution (with 6 degrees of freedom), and the green the matching Gaussian. 


\section{Appendix C: Model Checking}

The most important assumption of our models is the linearity of expected voxel activity as a function of stimulus features. If this holds, actual and predicted activities should themselves be linearly related. Figure 4 shows that this holds tolerably. In the absence of a neurobiologically-grounded alternative, or enough data to make nonparametric estimates practical, we thus stay with the reasonable, and computationally cheap, linear model.
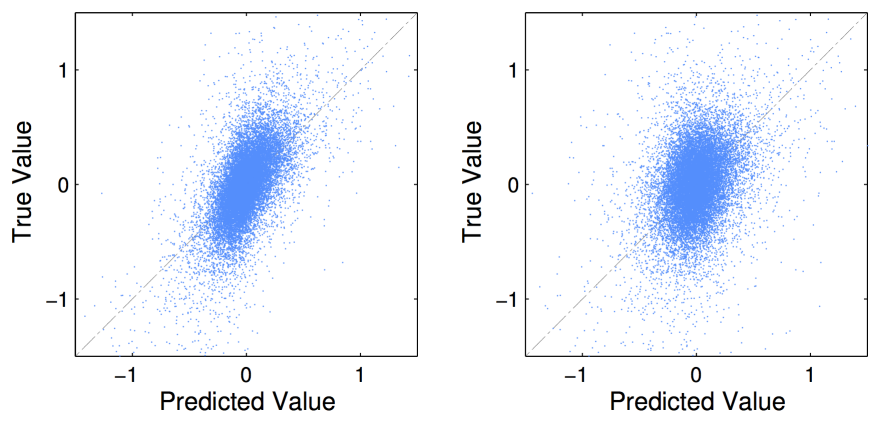

Fig 4: Scatter plots of true activity versus predicted activity using ridge regression for in-sample data (left) and out-of-sample data (right) for 1000 voxels picked at random from the set of voxels with good classification accuracy (greater than 60\%).

With the HB model, one must both check the behavior of the posterior distribution (as approximated by the output of the Gibbs sampler), and check the prior itself. The Gibbs sampler showed little change in either parameter estimates or predictive performance when varying the hyper-parameters $\alpha$ and $\beta$ over an order of magnitude. Posterior predictive simulations (Appendix C.1) indicated that if the SAE model was well specified it should out-predict ridge. Since this is not the case with the data, this suggests model misspecification.

\section{C.1. Simulation of the SAE Model}

App. B suggest that ridge regression and the SAE model should lead to very similar parameter estimates and hence to similar predictions. However, in our data we found the two to be virtually indistinguishable. Here, we show that this should not be the case if the SAE model were properly specified, and investigate what sort of mis-specification might account for it.

In these simulations, we simulate from the small-area model of $\S 2.3$, drawing parameters from the prior. For speed and simplicity, we limit ourselves to 500 voxels, divided into 5 ROIs of 100 voxels each. The stimuli $x$ were fixed to those employed in $\mathrm{E} 2$, and values of $y_{v}(t)$ draw conditional on $x$ and the randomlygenerated parameters. The surrogate values from this simulation were then fit to 
three models: ridge regression; the small-area model with the correct assignment of voxels to ROIs; and the small-area model with 100 voxels assigned to 5 ROIs at random.

Figures 5 and 6 show that the properly-specified small-area model has a modest, but systematic and significant, advantage in forward prediction over ridge regression. This disappears, however, when the small-area model is misspecified because it gets the assignment of voxels to areas wrong. As this predictive equivalence is more or less what we see in the data from both experiments, the left-hand panels of the figures lets us conclude that that the HB model is mis-specified "somehow". The right-hand panels suggest, but do not prove, that the mis-specification arises from using the wrong division of voxels into regions.
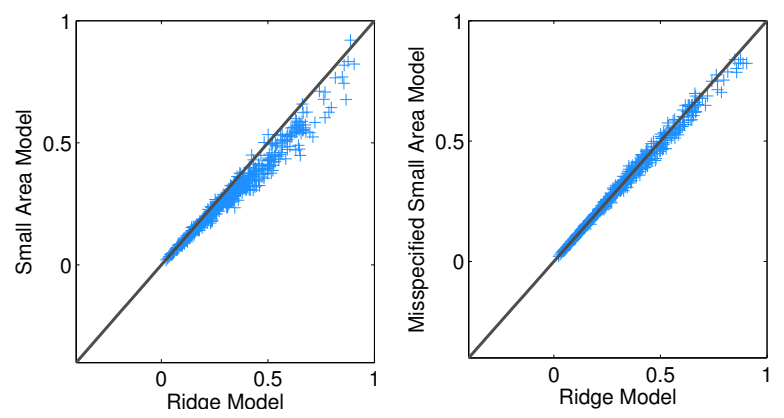

Fig 5: Voxel-wise RSS for the small-area model (vertical axis) versus that for ridge regression (horizontal), fit to simulations of the small-area model, with the assignment of voxels to ROIs being either correct (left) or incorrect (right). The small area estimates outperforms the ridge estimates when the true underlying model is a small area model and the small area estimator is correctly specified.
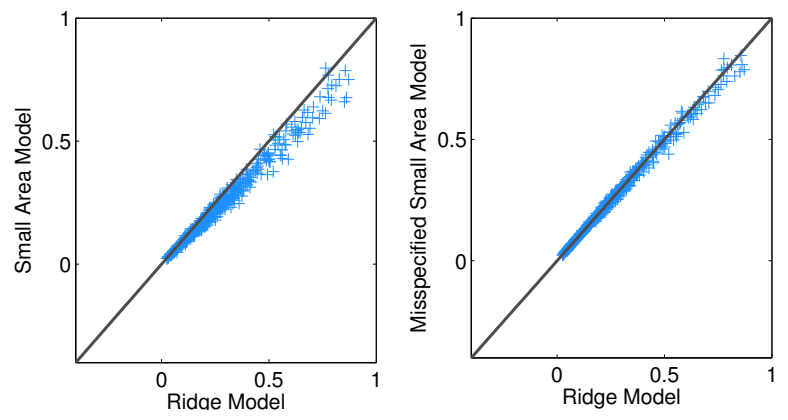

Fig 6: As in Figure 5, but increasing the extend to which shared area parameters vary between areas. Again, the small area estimates outperforms the ridge estimates when the true underlying model is a small area model and the small area estimator is correctly specified. 


\section{Appendix D: Regularization Reduces Variability}

Figure 7 shows how regularization, either by the ridge penalty or the prior of the SAE model, reduces standard errors in parameter estimates, compared to OLS. This indicates that the problem is one of small-area estimation in the technical sense, and needs some form of regularization.
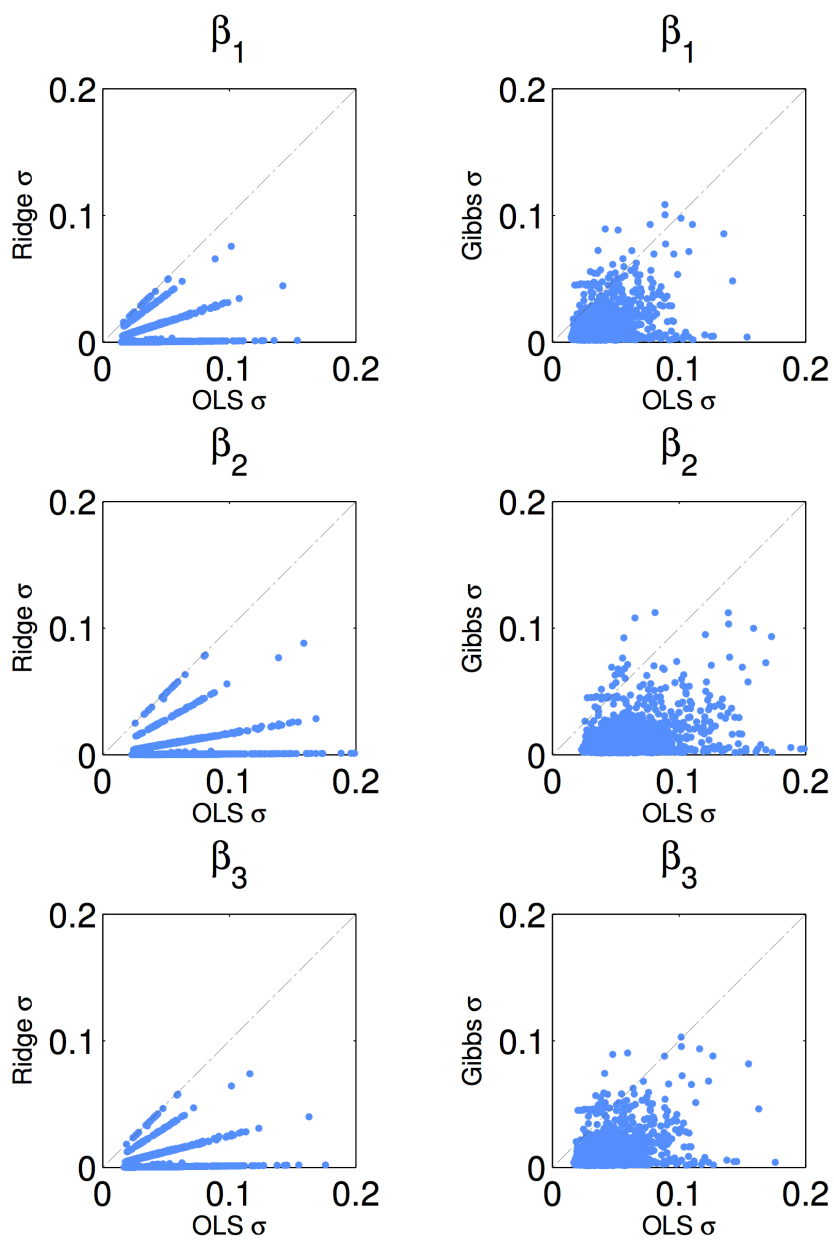

Fig 7: Standard errors of voxel-wise ridge-regression estimates (left column) and of SAEs (right), versus the standard errors of direct OLS estimates. Regularization (either with Ridge or SAE) reduces the standard errors of the estimates, indicating that the parameters need some form of regularization. 


\section{Appendix E: What is the effect of smoothing and regularization?}

To see the effect of smoothing and regularization on OLS we compared the held out normalized RSS before and after smoothing, and with and without regularization in $\S 3.1$. Here, we show the effect on the single voxel accuracies for both experiments. As seen in $\S 3.1$, smoothing seems to reduce the forward prediction error in the worst voxels, but its effects on classification accuracy are at best ambiguous (Figure 9). Much the same is true of shrinkage (Figure 8).
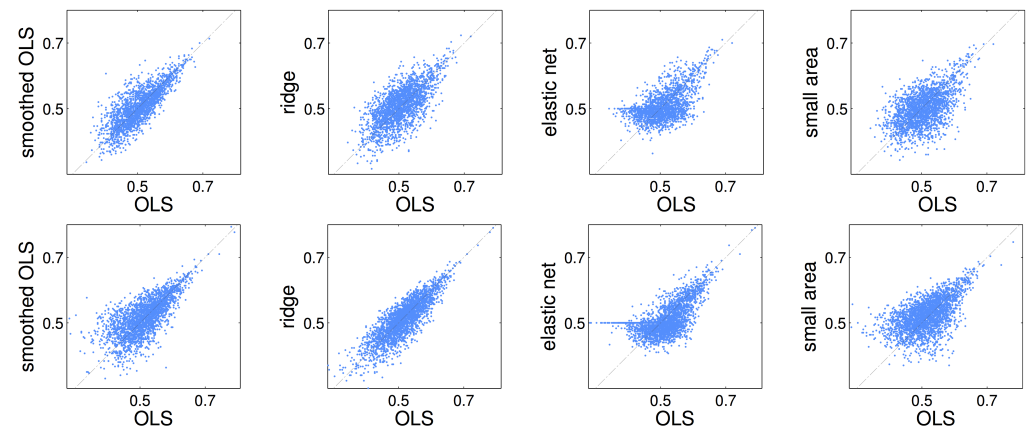

Fig 8: Effect of smoothing or shrinkage on voxel-wise classification accuracy for E1 (top) and E2 (bottom). For each of the plots, the OLS accuracy (horizontal axis) is contrasted with the modified accuracy after OLS smoothing or ridge, elastic net or small area shrinkage (vertical axis). For both experiments, is it hard to discern any systematic effect of smoothing or shrinkage in this reverse inference task.
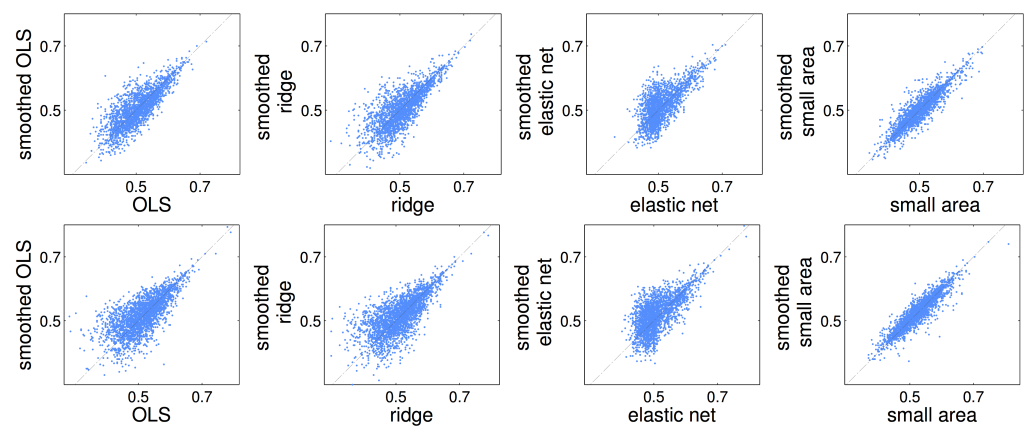

Fig 9: Effect of smoothing on voxel-wise classification accuracy for E1 (top) and E2 (bottom). For each of the plots, the unsmoothed OLS, ridge, elastic net or small area estimators (horizontal axis) are contrasted with their smoothed version (vertical axis). For both experiments, is it hard to discern any systematic effect of smoothing or shrinkage in this reverse inference task. 


\section{Appendix F: Full Brain results}

\section{F.1. Experiment 1 (E1)}

This section has 4 plots, which summarize our findings for the four methods of OLS, ridge regression, elastic net and the small area model for the first experiment (E1) described in Section 1.2. Color schemes are flipped so that red always represents "good" and blue, "bad". The images are best viewed on a computer screen with high resolution.

(A)

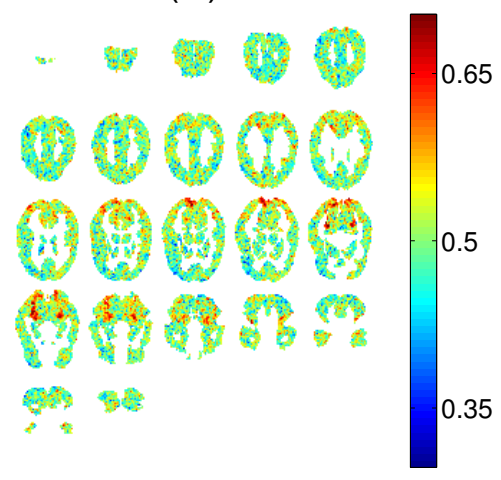

(C)

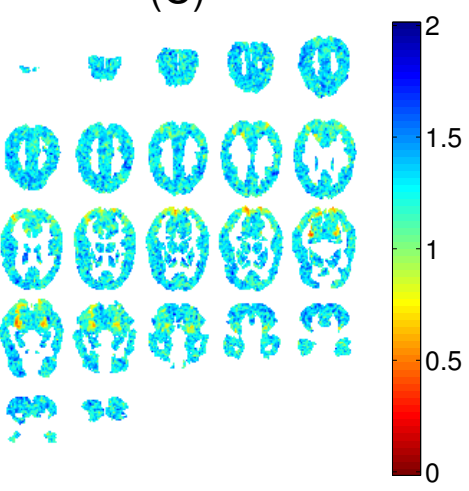

(B)

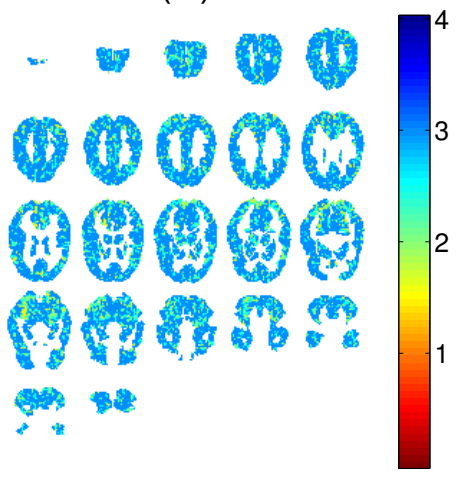

(D)

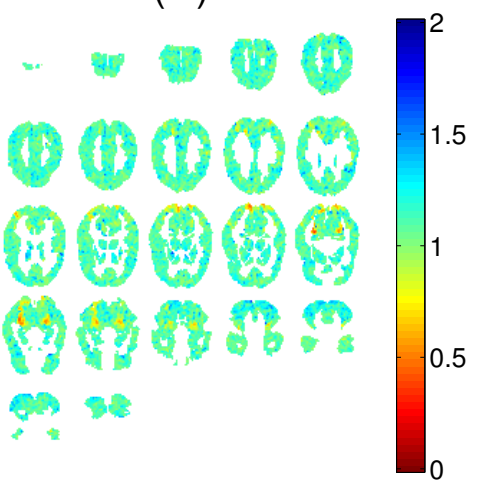

Fig 10: E1: OLS classification accuracy (A), smoothing radius (B) and normalized out-of-sample RSS before (C) and after smoothing (D). Note how predictive performance (sub-figures $\mathrm{A}$ and $\mathrm{D}$ ) is inversely related to the degree of regularization, which in this case is the is the smoothing radius for OLS. Note the improvement of the normalized out-of-sample RSS in the bad voxels after smoothing (indicated by less blue voxels in subplot D than subplot $\mathrm{C}$ ). 
(A)

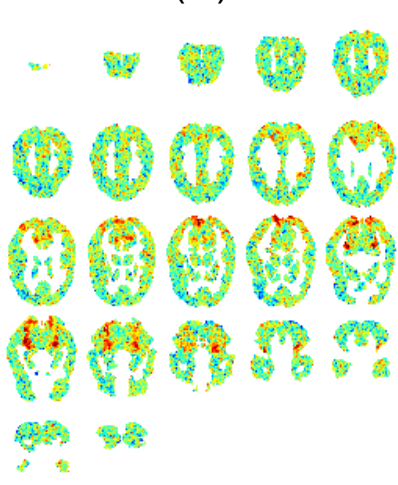

(B)
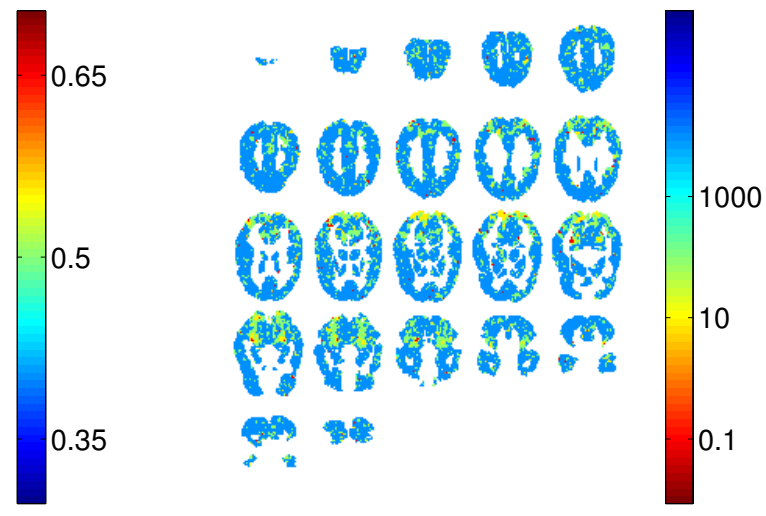

(D)

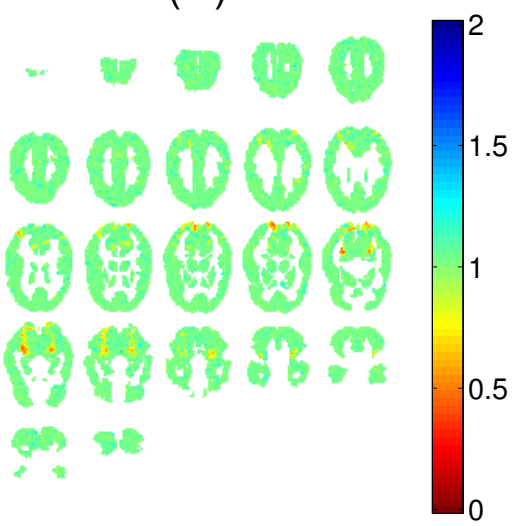

Fig 11: E1: ridge regression classification accuracy (A), $\lambda(B)$ and normalized RSS in (C) and out of sample (D). Note how predictive performance (sub-figures $\mathrm{A}$ and $\mathrm{D}$ ) is inversely related to the degree of regularization which in this case is the $\lambda$ penalty for ridge. Low regularization corresponds to accurate voxels, i.e., these "good" voxels are allowed to pick their parameters more freely. The in- and out-of- sample errors for "good" voxels are nearly the same. 
(A)

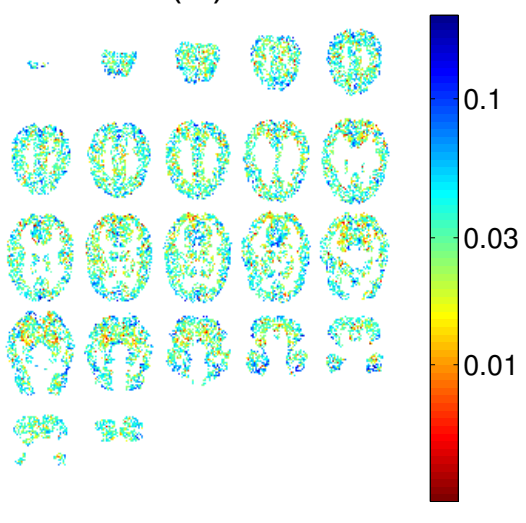

(C)

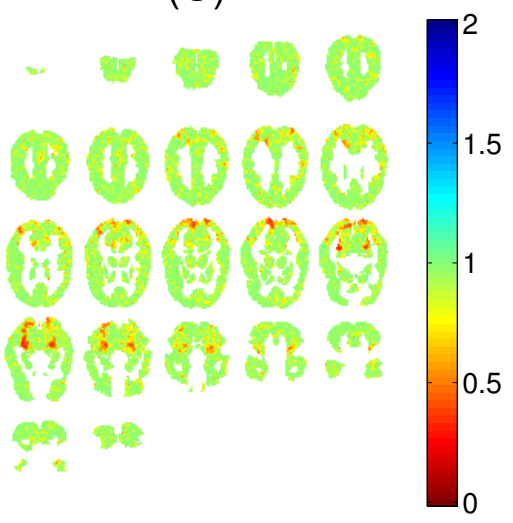

(B)

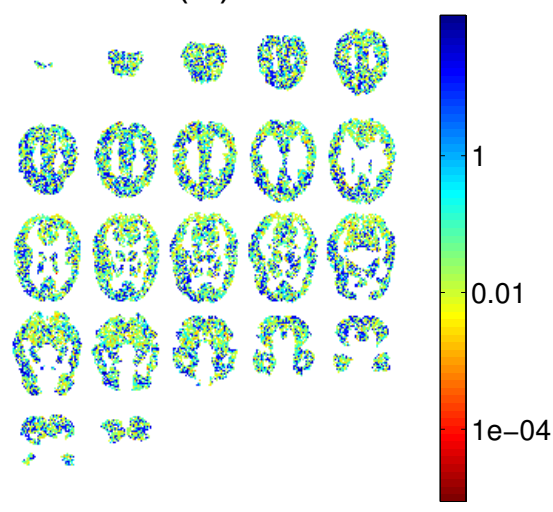

(D)

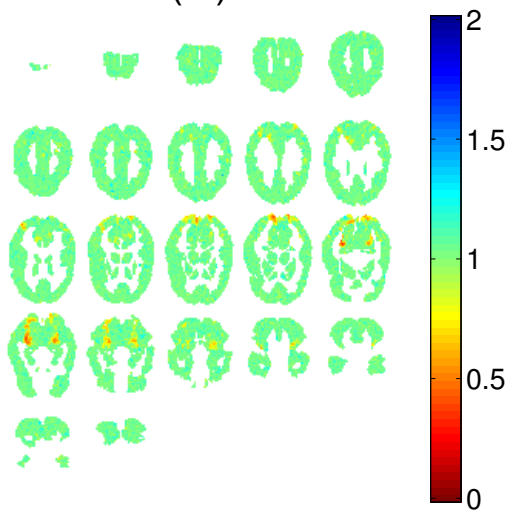

Fig 12: E1: Elastic net $\lambda_{1}$ (lasso penalty) (A), $\lambda_{2}$ (ridge penalty) (B) and normalized RSS in (C) and out of sample (D). Note how predictive performance (sub-figure D) is inversely related to the degree of regularization, which in this case is the $\lambda_{1}$ and $\lambda_{2}$ penalties for the elastic net. Good voxels have more lassolike penalties, as they are voxels sensitive to some of the stimulus features. The in- and out-of- sample errors for "good" voxels are nearly the same. 
(A)

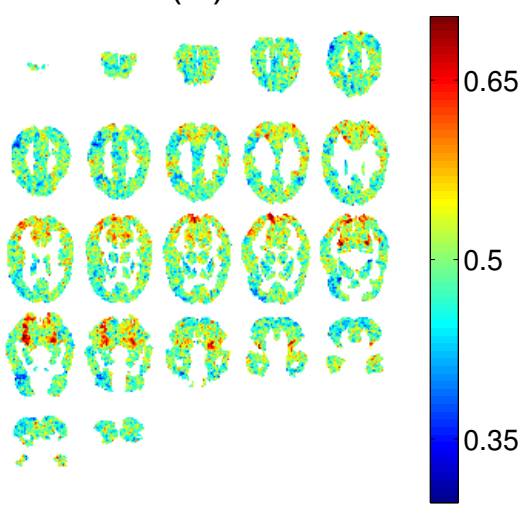

(C)

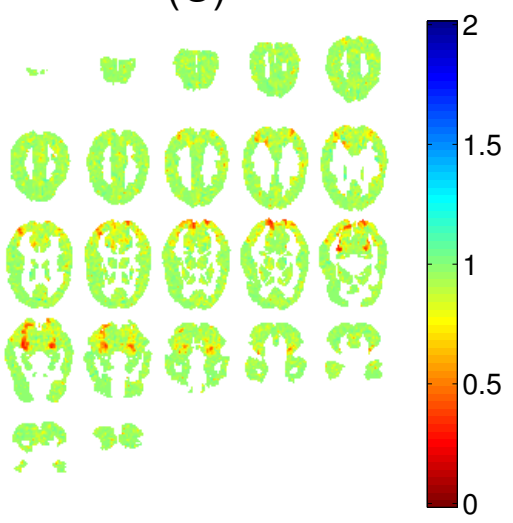

(B)

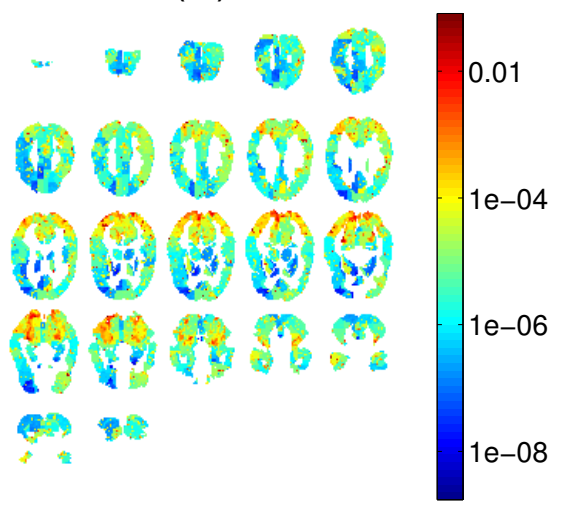

(D)

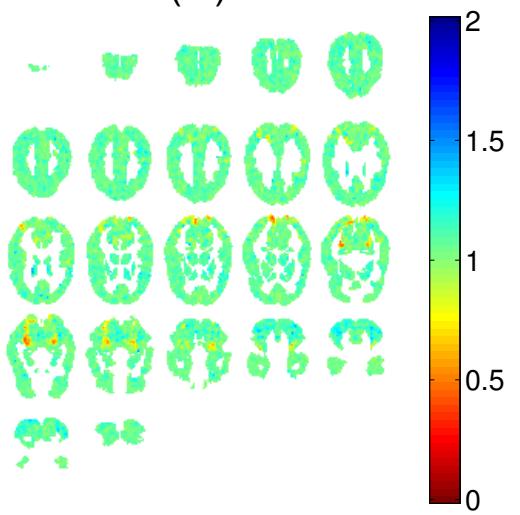

Fig 13: E1: small-area model classification accuracy (A), posterior mean of the variance of $\beta_{v}$ per voxel (B) and normalized RSS in (C) and out of sample (D). Note how predictive performance (sub-figures A and D) is inversely related to the degree of regularization of the small area model: low regularization corresponds to a high variance parameter, i.e., good voxels are allowed to pick their parameters freely. The in- and out-of- sample errors for "good" voxels are nearly the same. 


\section{F.2. Experiment 2 (E2)}

This section has 4 plots, which summarize our findings for the four methods of OLS, ridge regression, elastic net and the small area model for the second experiment (E2) described in Section 1.3. Color schemes are flipped so that red always represents "good" and blue, "bad". The images are best viewed on a computer screen with high resolution.

(A)

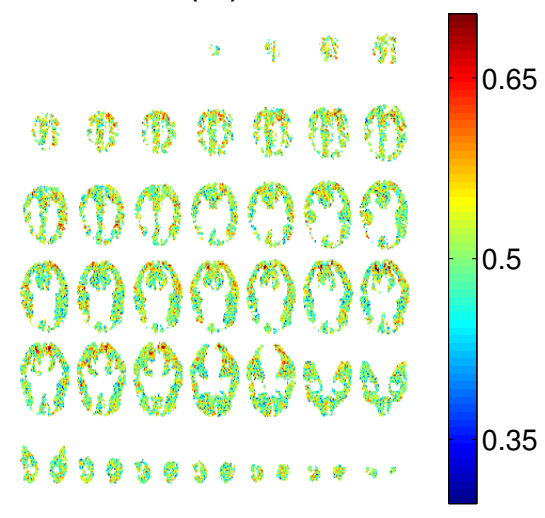

(C)

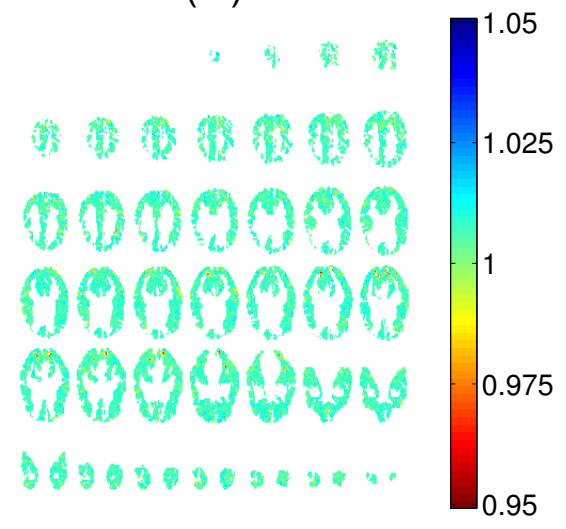

(B)

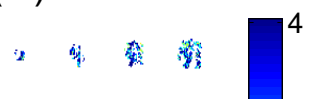

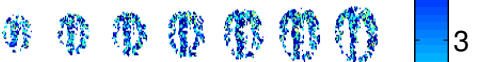

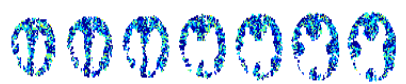

1)

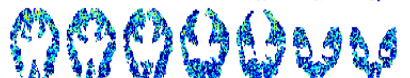

64.
(D)

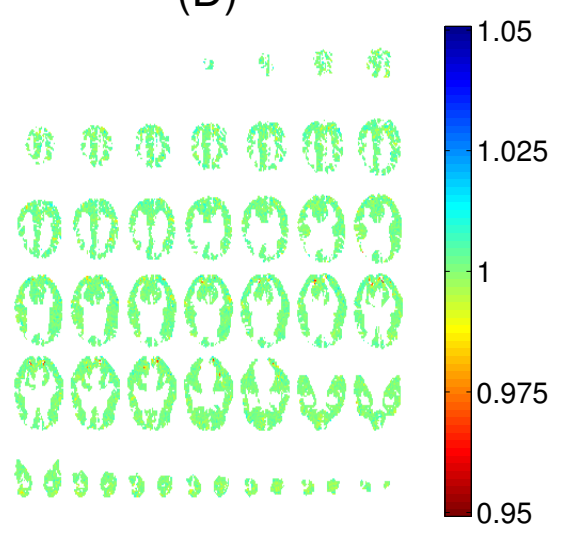

Fig 14: E2: OLS classification accuracy (A), smoothing radius (B) and normalized out-of-sample RSS before (C) and after smoothing (D). Note how predictive performance (sub-figures $\mathrm{A}$ and $\mathrm{D}$ ) is inversely related to the degree of regularization, which in this case is the is the smoothing radius for OLS. Note the improvement of the normalized out-of-sample RSS in the bad voxels after smoothing (indicated by less blue voxels in subplot D than subplot C). 
(A)

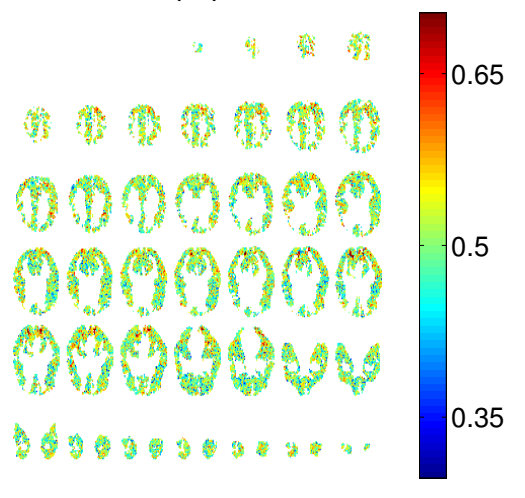

(C)

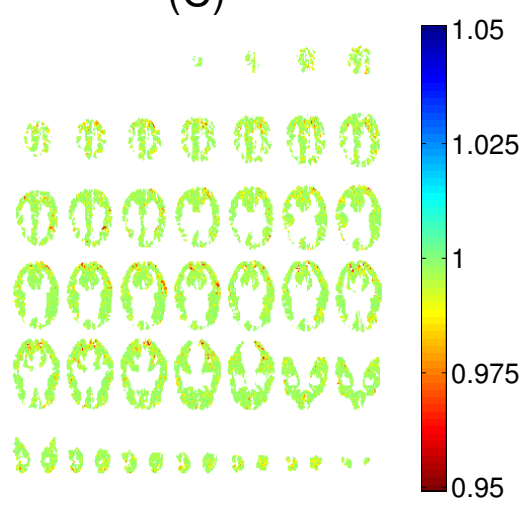

(B)

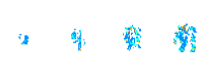

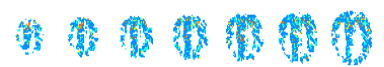

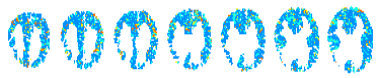

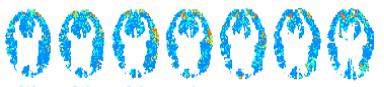

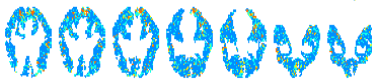

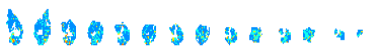

$-1000$

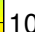

0.1
(D)

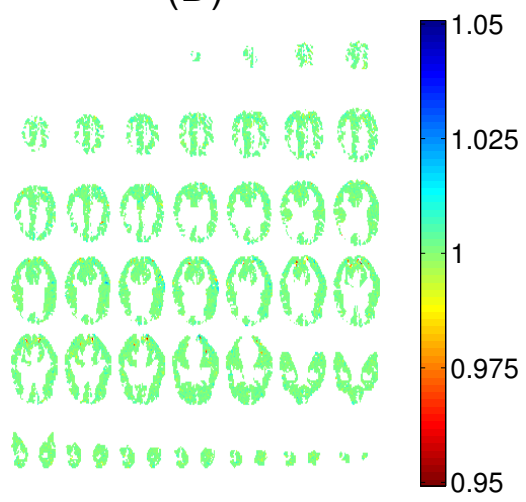

Fig 15: E2: ridge regression classification accuracy (A), $\lambda$ (B) and normalized RSS in (C) and out of sample (D). Note how predictive performance (sub-figures $\mathrm{A}$ and $\mathrm{D}$ ) is inversely related to the degree of regularization which in this case is the $\lambda$ penalty for ridge. Low regularization corresponds to accurate voxels, i.e., these "good" voxels are allowed to pick their parameters more freely. The in- and out-of- sample errors for "good" voxels are nearly the same. 
(A)

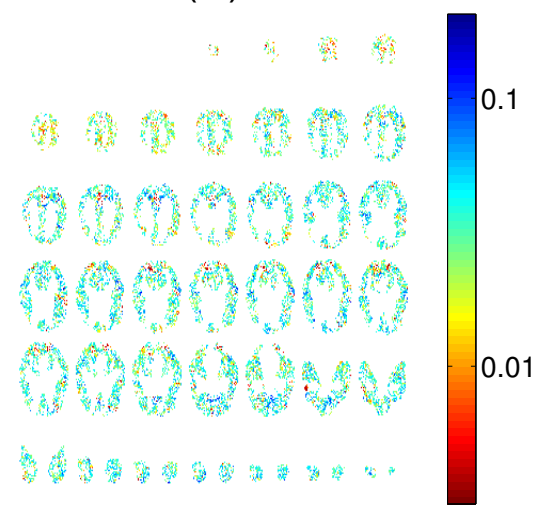

(C)

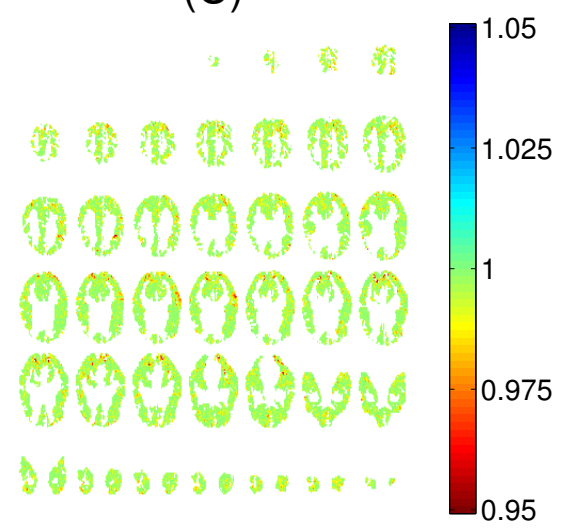

(B)

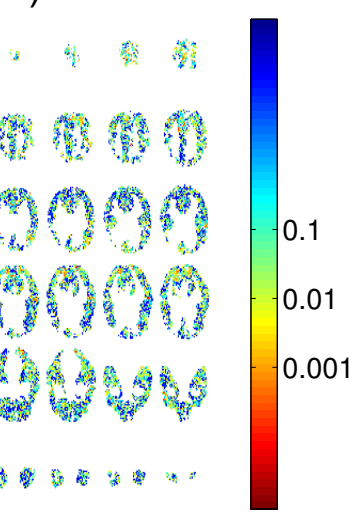

(D)

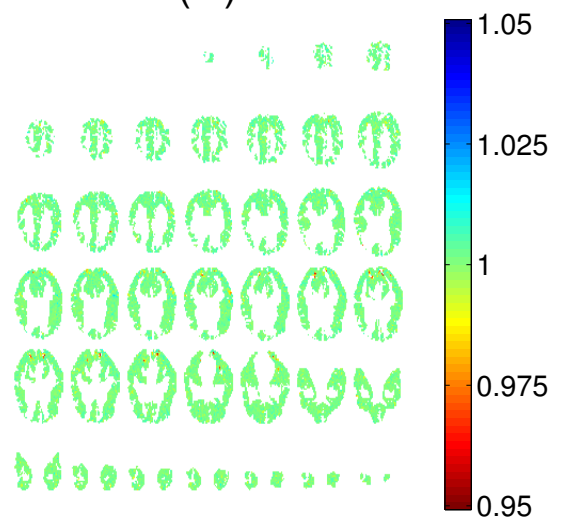

Fig 16: E2: Elastic net $\lambda_{1}$ (lasso penalty) (A), $\lambda_{2}$ (ridge penalty) (B) and normalized RSS in (C) and out of sample (D). Note how predictive performance (sub-figure D) is inversely related to the degree of regularization, which in this case is the $\lambda_{1}$ and $\lambda_{2}$ penalties for the elastic net. Good voxels have more lassolike penalties, as they are voxels sensitive to some of the stimulus features. The in- and out-of- sample errors for "good" voxels are nearly the same. 
(A)

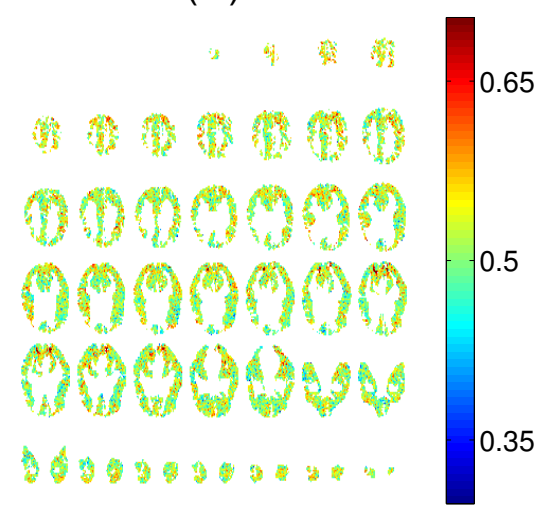

(C)

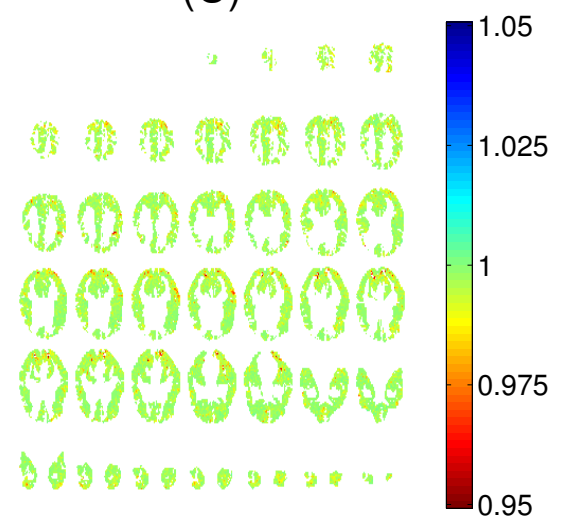

(B)

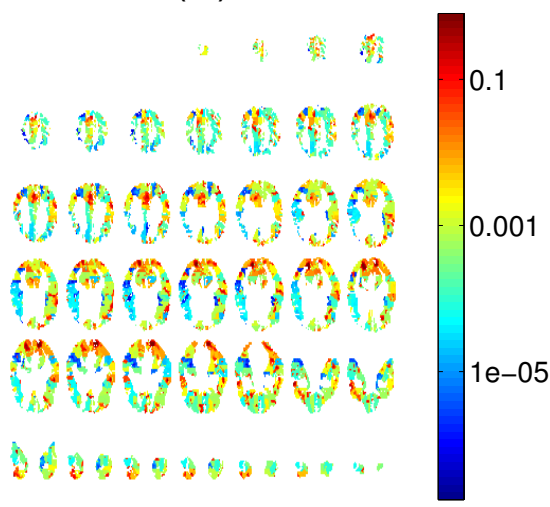

(D)

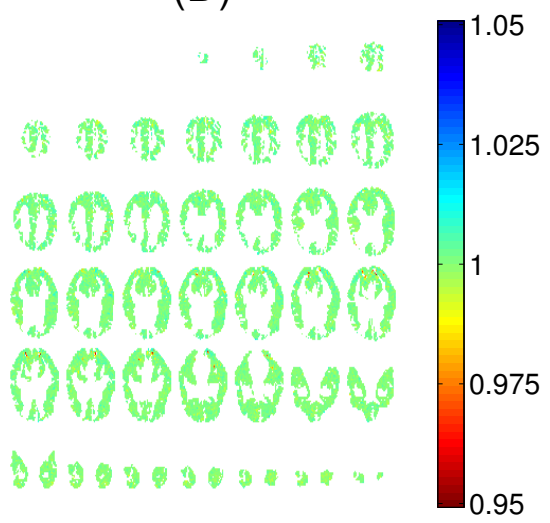

Fig 17: E2: small-area model classification accuracy (A), posterior mean of the variance of $\beta_{v}$ per voxel (B) and normalized RSS in (C) and out of sample (D). Note how predictive performance (sub-figures A and D) is inversely related to the degree of regularization of the small area model: low regularization corresponds to a high variance parameter, i.e., good voxels are allowed to pick their parameters freely. The in- and out-of- sample errors for "good" voxels are nearly the same. 